\title{
PICARD NUMBER OF THE GENERIC FIBER OF AN ABELIAN FIBERED HYPERKÄHLER MANIFOLD
}

\author{
KEIJI OGUISO
}

\begin{abstract}
We shall show that the Picard number of the generic fiber, in the sense of scheme, of an abelian fibered hyperkähler manifold over the projective space is always one. We then give a few applications for the Mordell-Weil group. In particular, by deforming O'Grady's 10-dimensional manifold, we construct an abelian fibered hyperkähler manifold of Mordell-Weil rank 20, which is the maximum possible among all known ones.
\end{abstract}

\section{INTRODUCTION}

By a hyperkähler manifold (HK manifold, for short), we mean a simply connected compact Kähler manifold $X$ admitting an everywhere non-degenerate holomorphic 2form $\sigma_{X}$ s.t. $H^{0}\left(X, \Omega_{X}^{2}\right)=\mathbf{C} \sigma_{X}$. Note that $X$ is even dimensional. Put $\operatorname{dim} X=2 n$. We call a surjective morphism $f: X \longrightarrow B$ a fibered $H K$ manifold if in addition $B$ is normal, projective and $f$ has connected fibers. A fundamental result of Matsushita ([M1], [M2]) says that each fiber of $f$ is Lagrangian. Especially smooth fibers are complex tori of dimension $n$. They are also projective so that abelian varieties (see eg. Og2). We call $f$ an abelian fibered $H K$ manifold if in addition $f$ admits a bimeromorphic section, i.e., a subvariety $O \subset X$ s.t. the induced morphism $f \mid O: O \longrightarrow B$ is bimeromorphic. Then, $X$ is projective (see eg. Og2 ) and we can work in the category of algebraic varieties over $\mathbf{C}$. In particular, we can speak of the generic fiber $X_{\eta}$ of $f$ in the sense of scheme. This is an abelian variety with origin $O \mid X_{\eta}$, defined over the function field $\mathbf{C}(B)$. Concerning the base space of a fibered HK manifold, there is a conjecture that it is always the projective space $\mathbf{P}^{n}$. Hwang $[\mathrm{Hw}]$ has shown that this is true if $X$ is projective and $B$ is smooth.

In this note, we study an abelian fibered HK manifold over the projective space. Our main results are Theorems 1.1, 1.2, 1.3 and 1.4.

Theorem 1.1. Let $f: X \longrightarrow \mathbf{P}^{n}$ be an abelian fibered $H K$ manifold. Let $K=\mathbf{C}\left(\mathbf{P}^{n}\right)$ and let $A_{K}$ be the generic fiber of $f$. Then, $\rho\left(A_{K}\right)=1$. Here $\rho\left(A_{K}\right)$ is the Picard number of $A_{K}$ over $K$.

2000 Mathematics Subject Classification. 14D06, 14J40, 14J28. 
There happens that $\rho\left(X_{t}\right) \geq 2$ for all smooth closed fiber of $f$ (see at the end of proof of Theorem $1.4(1))$. The statement is of arithmetical nature. Geometrically, it means that two horizontal divisors on $X$ are proportional in $N S(X)$ up to vertical divisors. Key of our proof is to compare two different ways of deformation of $f$; Matsushita's way [M3] and Voisin's way [Vo. The argument is very short, but it is quite mysterious, at least for me, that such a consideration yields a strong information about the Picard number of the generic fiber. It may be interesting, if possible, to give a more natural proof based on the monodromy representation around the discriminant locus $D \subset \mathbf{P}^{n}$, which is of pure codimension one $([\mathrm{HO}],[\mathrm{Hw}])$.

The set $A_{K}(K)$ of $K$-rational points, or equivalently, the set of rational sections of $f$, forms an abelian group called the Mordell-Weil group of $f$. We denote it by MW $(f)$. When MW $(f)$ is finitely generated, we call $m w(f):=\operatorname{rank} \mathrm{MW}(f)$ the Mordell-Weil rank. Mordell-Weil group and Mordell-Wel rank are important invariants of an abelian fibered variety. Combining Theorem 1.1 with [HPW] (or [Kh], Og2]), we obtain:

Theorem 1.2. Let $f: X \longrightarrow \mathbf{P}^{n}$ be as in Theorem 1.1. Let $D=\cup_{i=1}^{k} D_{i}$ be the irreducible decomposition of the discriminant divisor $D \subset \mathbf{P}^{n}$ and let $m_{i}$ be the number of prime divisors $(\subset X)$ lying over $D_{i}$. Then the Mordell-Weil group $\mathrm{MW}(f)$ is a finitely generated abelian group of rank

$$
m w(f)=\rho(X)-2-\sum_{i=1}^{k}\left(m_{i}-1\right) .
$$

In particular, $2 \leq \rho(X) \leq b_{2}(X)-2$ and $m w(f) \leq \rho(X)-2$.

Note that two inequalities in Theorem 1.2 are valid even if the base space would be singular $\mathrm{Og} 2$. It is natural to ask the optimality of these inequalities. This is also a problem of arithmetical nature, but again by geometry, we obtain the following:

Theorem 1.3. Let $f: X \longrightarrow \mathbf{P}^{n}$ be as in Theorem 1.1. Assume that $X$ admits at least one (not only rational but also) holomorphic section, i.e., a subvariety $O \subset X$ s.t. $f \mid O: O \longrightarrow \mathbf{P}^{n}$ is an isomorphism. Then, for each integer $\rho$ s.t. $2 \leq \rho \leq b_{2}(X)-2$, there is an abelian fibered $H K$ manifold $f_{\rho}: X_{\rho} \longrightarrow \mathbf{P}^{n}$ which is deformation equivalent to $X, \rho\left(X_{\rho}\right)=\rho$ and $m w\left(f_{\rho}\right)=\rho-2$. Moreover, for each $\rho$, such abelian fibered $H K$ manifolds are dense around $X$ in the Kuranishi space w.r.t. Euclidean topology.

This is a generalization of our earlier result Og1 about Mordell-Weil groups of Jacobian K3 surfaces (see also Og2 for an intermediate result).

As an application, we obtain: 
Theorem 1.4. (1) Let $\rho$ be an integer s.t. $2 \leq \rho \leq 21$ and let $n \geq 2$ be an integer. Then, for each such $\rho$ and $n$, there is an abelian fibered $H K$ manifold $f: X \longrightarrow \mathbf{P}^{n}$ s.t. $X$ is deformation equivalent to the Hilbert scheme $S^{[n]}$ of n-points of a K3 surface $S, \rho(X)=\rho$ and $m w(f)=\rho-2$. Note that $S^{[n]}$ is a HK manifold of $\operatorname{dim} S^{[n]}=2 n$ and $b_{2}\left(S^{[n]}\right)=23$ $[\mathrm{Be}]$.

(2) Let $\rho$ be an integer s.t. $2 \leq \rho \leq 22$. Then, for each such $\rho$, there is an abelian fibered $H K$ manifold $f: X \longrightarrow \mathbf{P}^{5}$ s.t. $X$ is deformation equivalent to O'Grady's 10dimensional $H K$ manifold $\mathcal{M}_{4}\left[\mathrm{OGr}, \rho(X)=\rho\right.$ and $m w(f)=\rho-2$. Note that $\mathcal{M}_{4}$ is a $H K$ manifold with largest known second Betti number, $b_{2}\left(\mathcal{M}_{4}\right)=24$ [Ra2]. In particular, the case $\rho=22$ gives the largest record of the Mordell-Weil rank 20, among all the currently known abelian fibered HK manifolds.

We show Theorems 1.1, 1.2 in Section 2 and Theorems 1.3, 1.4 in Section 3.

\section{Proof of Theorems 1.1 and 1.2}

Let $\mathcal{P}=\left\{[\sigma] \in \mathbf{P}\left(H^{2}(X, \mathbf{C})\right) \mid(\sigma, \sigma)=0,(\sigma, \bar{\sigma})>0\right\}$ be the period domain of $X$. By the local Torelli theorem for HK manifolds (see eg. [GHJ] Proposition 22.11), we can (and will) identify the Kuranishi space $\mathcal{K}$ of $X$ with a small neighbourhood of $\left[\sigma_{X}\right] \in \mathcal{P}$, which we also write by $\mathcal{P} ; \mathcal{K}=\mathcal{P}$. As we consider a small neighbourhood of $\left[\sigma_{X}\right] \in \mathcal{P}$ only, no confusion occurs.

Let $F$ be a smooth closed fiber of $f$ and let $\iota: F \longrightarrow X$ be the inclusion map. As $f$ is fibered over $\mathbf{P}^{n}$, the deformation of $X$ that keeps fibration is of codimension 1 in $\mathcal{P}$ by Matsushita [M3] Corollary 1.7 (see also [Sa2]). More precisely, it is a hypersurface defined by $(h, \sigma)=0$, where $h=f^{*} H$ is the pullback of the hyperplane class $H$ of $\mathbf{P}^{n}$. Here the fact that the base space is $\mathbf{P}^{n}$ is important. As fibers of a fibered HK manifold are Lagrangian [M2], this deformation is a (part of) deformation of $X$ that keeps $F$ Lagrangian. By Voisin [Vo] (an easier direction), the latter space is of codimension $r$ in $\mathcal{P}$, where $r=\operatorname{rank} \operatorname{Im}\left(\iota^{*}: H^{2}(X, \mathbf{Z}) \longrightarrow H^{2}(F, \mathbf{Z})\right)$. Thus we have $r=1$.

Let us show that $\rho\left(A_{K}\right)=1$. For this, it suffices to show that two elements $L_{1}$ and $L_{2}$ of $N S\left(A_{K}\right)$ are proportional. As $A_{K}$ is projective, we may (and will) assume that both are represented by very ample divisors on $A_{K}$. We can write $L_{i}=\left[H_{i}\right] \mid A_{K}(i=1,2)$. Here $H_{i}(i=1,2)$ are effective Cartier divisors on $X$ and $\left[H_{i}\right] \in N S(X)$ are their classes.

Let $U \subset \mathbf{P}^{n}$ be a dense Zariski open subset of $\mathbf{P}^{n}$ s.t. $f$ is smooth over $U$ and both $H_{i}$ are flat over $U$. Let $X_{U}=f^{-1}(U)$. Consider the morphism $\iota: \operatorname{Div}\left(X_{U} / U\right) \longrightarrow$ $\operatorname{Pic}\left(X_{U} / U\right)$ over $U$. (For the Picard scheme, see [Gr] or [FK], Chapter 9.) 
Let $F$ be a general closed fiber of $f$ over $t \in U$. The two classes $\iota_{t}\left(H_{i} \mid F\right)(i=1,2)$ are linearly dependent in $N S(F)$ by $r=1$. Thus there is a pair of positive integers $\left(n_{1}, n_{2}\right)$ s.t. $\iota_{t}\left(n_{1} H_{1} \mid F\right)-\iota_{t}\left(n_{2} H_{2} \mid F\right) \in \operatorname{Pic}^{0}(F)$.

As both $\operatorname{Pic}\left(X_{U} / U\right)$ and its identity component $\operatorname{Pic}^{0}\left(X_{U} / U\right)$ satisfy the base change property, it follows that $\iota\left(n_{1} H_{1}\right)-\iota\left(n_{2} H_{2}\right) \in \operatorname{Pic}^{0}\left(X_{U} / U\right)$. As $U$ contains the generic point $\eta$ of $\mathbf{P}^{n}$, we have then $\iota_{\eta}\left(n_{1} H_{1} \mid A_{K}\right)-\iota_{\eta}\left(n_{2} H_{2} \mid A_{K}\right) \in \operatorname{Pic}^{0}\left(A_{K}\right)$, again by the base change property. Thus, $n_{1} L_{1}=n_{2} L_{2}$ in $N S\left(A_{K}\right)$. This completes the proof of Theorem 1.1 .

Substituting $\rho\left(\mathbf{P}^{n}\right)=\rho\left(A_{K}\right)=1$ into the formula Og2 Theorem 1.1 (or [HPW] Proposition 2.6 and Lemma 2.4, or [Kh] ), we obtain Theorem 1.2 .

\section{Proof of Theorems 1.3 and 1.4}

Let $f: X \longrightarrow \mathbf{P}^{n}$ and $O \simeq \mathbf{P}^{n}$ be as in Theorem 1.3 and let $F$ be a general closed fiber of $f$. Then $O$ and $F$ are Lagrangian submanifolds of $X$. Consider the deformation $\mathcal{X} \longrightarrow \mathcal{B} \subset \mathcal{P}$ of $f: X \longrightarrow \mathbf{P}^{n}$ which keeps $F$ and $O$ Lagrangian. (Here, we denote by the same letter $\mathcal{P}$ a small neighbourhood of $\left[\sigma_{X}\right]$ in the period domain $\mathcal{P}$ and identify it with the Kuranishi space $\mathcal{K}$ of $X ; \mathcal{P}=\mathcal{K}$, as in Section 2.) Let $\left[\sigma_{X}\right] \in U \subset \mathcal{P}$ be a small neighbourhood of $\left[\sigma_{X}\right]$.

By [Sa2] (see also Og2 Proof of Proposition 3.3), the deformation $\mathcal{X} \longrightarrow \mathcal{B}$ forms a projective family of abelian fibered HK manifolds $\tilde{f}: \mathcal{X} \longrightarrow \mathbf{P}^{n} \times \mathcal{B}$, over $\mathcal{B}$, with holomorphic section $\mathcal{O} \simeq \mathbf{P}^{n} \times \mathcal{B}$. The base space $\mathcal{B}$ is a complex submanifold of codimension 2 in $\mathcal{P}$, of the form $(a, \sigma)=(s, \sigma)=0$, where $a$ and $s$ are linearly independent elements in $H^{2}(X, \mathbf{Q})$. For this, we need the harder direction of [Vo] and the fact that $O \simeq \mathbf{P}^{n}$.

Let $\tilde{f}_{b}: \mathcal{X}_{b} \longrightarrow \mathbf{P}^{n}(b \in \mathcal{B})$ be the fibration induced by $\tilde{f}$. By the shape of equation of $\mathcal{B}$ and by the Lefschetz (1,1)-Theorem, we see that $\rho\left(\mathcal{X}_{b}\right) \geq 2$ for all $b \in \mathcal{B}$ and $\rho\left(\mathcal{X}_{b}\right)=2$ for generic $b \in \mathcal{B}$. Here, the word "generic" means that the condition is satisfied outside a countably many union of hypersurfaces. In fact, $\rho\left(\mathcal{X}_{b}\right) \geq \rho$ if and only if $\operatorname{dim}\left\{\ell \in H^{2}(X, \mathbf{Q}) \mid\left(\ell,\left[\omega_{\mathcal{X}_{b}}\right]\right)=0\right\} \geq \rho$, i.e., there are at least $\rho$ linearly independent rational hyperplanes passing through the point $\left[\sigma_{\mathcal{X}_{b}}\right]$ corresponding to $\mathcal{X}_{b}$. Thus, we have in fact a much stronger statement; in any open neighbourhood of $\left[\sigma_{X}\right] \in \mathcal{B}$, the subset $\left\{b \mid \rho\left(\mathcal{X}_{b}\right)=\rho\right\}$ is also dense w.r.t. Euclidean topology, for each integer $\rho$ s.t. $2 \leq \rho \leq b_{2}(X)-2$. (See Og1 Theorem 1.1 and its proof for more details).

Let $\mathcal{D} \subset \mathbf{P}^{n} \times \mathcal{B}$ be the discriminant locus of $\tilde{f}$. The discriminant locus of $\tilde{f}_{b}(b \in \mathcal{B})$ is then $\mathcal{D}_{b}=\mathcal{D} \cap\left(\mathbf{P}^{n} \times\{b\}\right)$. This is of pure codimension 1 in $\mathbf{P}^{n}$ ([HO] Proposition $3.1(2)$, $\left[\mathrm{Hw}\right.$ ] Proposition 4.1). Let $k(b)$ be the number of the irreducible components of $\mathcal{D}_{b}\left(\subset \mathbf{P}^{n}\right)$. Then $k(b)$ is upper-semicontinuous on $\mathcal{B}$ w.r.t. Zariski topology. Choose $p \in \mathcal{B} \cap U$ s.t. 
$k(p)$ is minimum, say $k$. There is then an open neighbourhood $p \in V \subset \mathcal{B} \cap U$ s.t. the number of irreducible components of $\mathcal{D}_{b}(b \in V)$ is $k$, in particular, constant. For the argument from now, we may (and will) assume that the irreducible decomposition of $\mathcal{D}$ is $\mathcal{D}=\cup_{i=1}^{k} \mathcal{D}_{i}$ and $\mathcal{D}_{b}=\cup_{i=1}^{k} \mathcal{D}_{i, b}$ is the irreducible decomposition of $\mathcal{D}_{b}(b \in V)$.

Take one general reference point $o \in V$ s.t. $\rho\left(\mathcal{X}_{o}\right)=2$. Then, by Theorem $1.2, \tilde{f}_{o}^{-1}\left(\mathcal{D}_{i, o}\right)$ are all irreducible. Moreover, they are reduced as $\tilde{f}$ admits a holomorphic section $\mathcal{O}$. Take then a resolution of singularities $\tilde{\mathcal{D}}_{i}$ of $\tilde{f}^{-1}\left(\mathcal{D}_{i}\right)$ and denote by $g: \tilde{\mathcal{D}}_{i} \longrightarrow V$ the natural morphism. Recall that smoothness of fibers is non-empty open condition w.r.t. Zariski topology for a proper morphism from a smooth variety (in characteristic 0). Then, by the generality of $o$ and by the fact that $\tilde{f}_{o}^{-1}\left(\mathcal{D}_{i, o}\right)$ is irreducible and reduced, we see that $g^{-1}(o)$ is also irreducible and smooth, and the same holds over some Zariski open neighbourhood $W$ of $o$. Thus $\tilde{f}_{b}^{-1}\left(\mathcal{D}_{i, b}\right)$ are irreducible and reduced for all $b \in W$. Thus, from Theorem 1.2, we have $m w\left(\tilde{f}_{b}\right)=\rho\left(\mathcal{X}_{b}\right)-2$ for all $b \in W$. On the other hand, as we already remarked, the set $W_{\rho}=\left\{b \in W \mid \rho\left(\mathcal{X}_{b}\right)=\rho\right\}$ is dense in $W$ (w.r.t. Euclidean topology) for each $\rho$ with $2 \leq \rho \leq b_{2}(X)-2$. Hence, for each such $\rho$, there is $b \in W \subset U$ s.t. $\rho\left(\mathcal{X}_{b}\right)=\rho$ and $m w\left(\tilde{f}_{b}\right)=\rho-2$. This completes the proof of Theorem 1.3 .

Let us show Theorem 1.4. Let $f: S \longrightarrow \mathbf{P}^{1}$ be an elliptic K3 surface with a section. Then $f$ induces an abelian fibration $\varphi: S^{[n]} \longrightarrow \mathbf{P}^{n}$ with holomorphic section. Thus, Theorem 1.4 (1) follows from Theorem 1.3. Note that $\rho(F) \geq 2$ for each smooth close fiber $F$ of $\varphi$, as $F$ is the product of elliptic curves (cf. Remark after Theorem 1.1).

For Theorem 1.4 (2), it suffices to find one abelian HK manifold $f: X \longrightarrow \mathbf{P}^{5}$ s.t. $X$ is deformation equivalent to O'Grady's 10-dimensional HK manifold $\mathcal{M}_{4}$ and $f$ admits a holomorphic section.

Let $S$ be a generic algebraic K3 surface of degree 2. Then, $\operatorname{Pic} S=\mathbf{Z} H, H$ is ample, and $\left(H^{2}\right)=2$. We denote by $\pi: S \longrightarrow \mathbf{P}^{2}$ the finite double cover induced by $|H|$. Let $\bar{Y}:=M((0,[2 H], 2))$ be the moduli space of semi-stable sheaves with Mukai vector $(0,[2 H], 2)$ on $S$. The space $\bar{Y}$ is singular, but it admits a symplectic resolution $Y$ [LS]. This $Y$ is a HK manifold and is birational to $\mathcal{M}_{4}$ ([OGr] Section 4). Hence $Y$ is deformation equivalent to $\mathcal{M}_{4}$ by a fundamental result of Huybrechts [Hu]. For each $I \in M((0,[2 H], 2))$, the fitting ideal of $I$ is the ideal sheaf of some $C \in|2 H|$ and vice versa ([Ra1] Remark 2.1.1. See also [Sa1]). Thus, we have a natural surjective morphism $\bar{g}: \bar{Y} \longrightarrow \mathbf{P}^{5} \simeq|2 H|$. Let $U \subset|2 H|$ be the open subset consisting of smooth members. If $[C] \in U$, then $C$ is a smooth curve of genus 5 and the fiber $\bar{g}^{-1}([C])$ is isomorphic $\operatorname{Pic}^{6}(C)$, which is an abelian variety of dimension 5 . The same is true for the induced fibration $g: Y \longrightarrow \mathbf{P}^{5} \simeq|2 H|$. As $C$ admits unique $g_{2}^{1}(C)$ corresponding to the double cover $\pi \mid C$, the map defined by $C \mapsto 3 g_{2}^{1}(C) \in \operatorname{Pic}^{6}(C)$ gives a section of $g$ over $U$. 
However, it is unclear if this section will be extended to a holomorphic section of $g$. The following idea to replace $Y$ further is due to Yasunari Nagai.

Similarly, one has the moduli space $\bar{X}:=M((0,[2 H],-4))$ of semi-stable sheaves with Mukai vector $(0,[2 H],-4)$ on $S$ and its symplectic resolution $X[\mathrm{LS}]$. For the same reason as before, we have a fibration $\bar{f}: \bar{X} \longrightarrow \mathbf{P}^{5} \simeq|2 H|$, and the induced fibration $f: X \longrightarrow \mathbf{P}^{5} \simeq|2 H|$. The fiber over $[C] \in U$ is $\operatorname{Pic}^{0}(C)$ for both $\bar{f}$ and $f$. As $g$ admits a section over $U$, we have $f^{-1}(U) \simeq g^{-1}(U)$ over $U$. Thus, $X$ is birational to $Y$, whence, to $\mathcal{M}_{4}$. So, $X$ is also deformation equivalent to $\mathcal{M}_{4}$ again by [Hu]. Moreover, the map $C \mapsto \mathcal{O}_{C}$ gives a holomorphic section $s: \mathbf{P}^{5} \longrightarrow P \subset \bar{X}$ of $\bar{f}$. On the other hand, by [LS], the singular locus $B$ of $\bar{X}$ consists of sheaves of the form $F_{1} \oplus F_{2}$. Therefore $P \cap B=\emptyset$. As $X$ is just a blow-up of $\bar{X}$ along $B$ [LS], it follows that $s$ gives rise to a holomorphic section of $f: X \longrightarrow \mathbf{P}^{5}$. Now, Theorem 1.4 (2) follows from Theorem 1.3 .

Acknowledgement. First of all, I would like to express my best thanks to Doctor Yasunari Nagai for his brilliant idea in the last step of the proof of Theorem 1.4 (2). I would like to express my thanks to Professors F. Catanese, Y. Kawamata, G. Tian and E. Viehweg for their invitation to Oberwolfach September 2007. An initial idea of this work was found during preparation of my talk requested by Professor F. Catanese. I express my thanks to Professors J.H. Keum and J.M. Hwang for invitation to KIAS. This work was completed during my stay there February 25-April 122008.

\section{REFERENCES}

[Be] A. Beauville, Variétés Kähleriennes dont la premiére classe de Chern est nulle, J. Differential Geom. 18 (1983) 755-782.

[FK] B. Fantechi, L. Göttsche, L. Illusie, S.L. Kleiman, N. Nitsure, A. Vistoli, Fundamental algebraic geometry. Grothendiecks FGA explained, Mathematical Surveys and Monographs, 123, American Mathematical Society, Providence, RI, 2005.

[GHJ] M. Gross, D. Huybrechts, D. Joyce, Calabi-Yau manifolds and related geometries, Lectures from the Summer School held in Nordfjordeid, June 2001. Universitext. Springer-Verlag, Berlin, 2003.

[Gr] A. Grothendieck, Fondements de la gëométrie algébrique, Secrtariat mathématique, Paris 1962.

[HPW] M. Hindry, A. Pacheco, and R. Wazir, Fibrations et conjecture de Tate, J. Number Theory 112 (2005) 345-368.

[Hu] D. Huybrechts, Compact hyperkahler manifolds: basic results, Invent. Math. 135 (1999) 63-113. Erratum: Compact hyperkahler manifolds: basic results, Invent. Math. 152 (2003) 209-212.

[Hw] J.M. Hwang, Base manifolds for fibrations of projective irreducible symplectic manifolds, arXiv:0711.3224.

[HO] J.M. Hwang and K. Oguiso, Characteristic foliation on the discriminantal hypersurface of a holomorphic Lagrangian fibration, arXiv:0710.2376.

[Kh] B. Kahn, Démonstration géométrque du Théoréme de Lang-Néron, math.AG/0703063.

[LS] M. Lehn, Manfred and C. Sorger, La singularit de O'Grady, J. Algebraic Geom. 15 (2006) 753-770. 
[M1] D. Matsushita, On fibre space structures of a projective irreducible symplectic manifold, Topology 38 (1999) 79-83. Addendum: On fibre space structures of a projective irreducible symplectic manifold, Topology 40 (2001) 431-432.

[M2] D. Matsushita, Equidimensionality of Lagrangian fibrations on holomorphic symplectic manifolds, Math. Res. Lett. 7 (2000), 389-391.

[M3] D. Matsushita, Higher direct images of dualizing sheaves of Lagrangian fibrations, Amer. J. Math. 127 (2005) 243-259.

[OGr] O'Grady, Desingularized moduli spaces of sheaves on a K3, J. reine angew. Math. 5121999 49-117.

[Og1] K. Oguiso, Local families of K3 surfaces and applications, J. Algebraic Geom. 12 (2003) 405-433.

[Og2] K. Oguiso, Shioda-Tate formula for an abelian fibered variety and applications, mathAG/0703245, to appear in Korean J. Math.

[Ra1] A. Rapagnetta, Topological invariants of O'Grady's six dimensional irreducible symplectic variety, Math. Z. 256 (2007) 1-34.

[Ra2] A. Rapagnetta, On the Beauville form of the known irreducible symplectic varieties, Math. Ann. 340 (2008) 77-95.

[Sa1] J. Sawon, Abelian fibred holomorphic symplectic manifolds, Turkish J. Math. 27 (2003) 197-230.

[Sa2] J. Sawon, Deformations of holomorphic Lagrangian fibrations, math.AG/0509223.

[Vo] C. Voisin, Sur la stabilité des sous-variete lagrangiennes des variété symplectiques holomorphes, Complex projective geometry, London Math. Soc. Lecture Note Ser. 179 (1992) 294-303, Cambridge Univ. Press, Cambridge.

Department of Economy, Keio University, Hiyoshi Hokuku-ku Yokohama, Japan, and Korea Institute for Advanced Study, 207-43 Cheonryangni-2dong, Dongdaemun-gu, Seoul 130-722, Korea

E-mail address: oguiso@hc.cc.keio.ac.jp 